\title{
Local structural fluctuations, hydrogen bonding and structural transitions in supercritical water.
}

\author{
Ioannis Skarmoutsos ${ }^{a^{*}}$, Elvira Guardia ${ }^{b}$ and Jannis Samios ${ }^{c}$ \\ ${ }^{a}$ Institut Charles Gerhardt Montpellier, UMR 5253 CNRS, Université de Montpellier, Place E. \\ Bataillon, 34095 Montpellier Cedex 05, France \\ *Corresponding Author: iskarmoutsos@hotmail.com \\ ${ }^{\mathrm{b}}$ Departament de Física, Universitat Politècnica de Catalunya, Campus Nord-Edifici B4-B5, Jordi \\ Girona 1-3, Barcelona E-08034, Spain \\ ${ }^{\mathrm{c}}$ National \& Kapodistrian University of Athens, Department of Chemistry, Laboratory of Physical \\ Chemistry, Panepistimiopolis, 157-71, Athens, Greece
}

\begin{abstract}
The contribution of hydrogen bonding interactions to the formation of local density inhomogeneities in supercritical water at near-critical conditions has been extensively studied by means of molecular dynamics simulations. The results obtained have revealed the strong effect of water molecules forming one and two hydrogen bonds on the determination of the local density augmentation in the fluid. The local structural order has also been studied in terms of the trigonal and tetrahedral order parameters, revealing the correlation between local orientational order and hydrogen bonding. The dynamics of the structural order parameters exhibit similarities with local density ones. The local structural analysis performed in terms of nearest neighbors around the individual molecules provides additional significant evidence about the existence of a liquid-like to gas-like structural transition in supercritical water at the density range close to $0.2 \rho_{c}$, further supporting previous suggestions based on the interpretation of experimental thermodynamic data.
\end{abstract}




\section{Graphical Abstract}

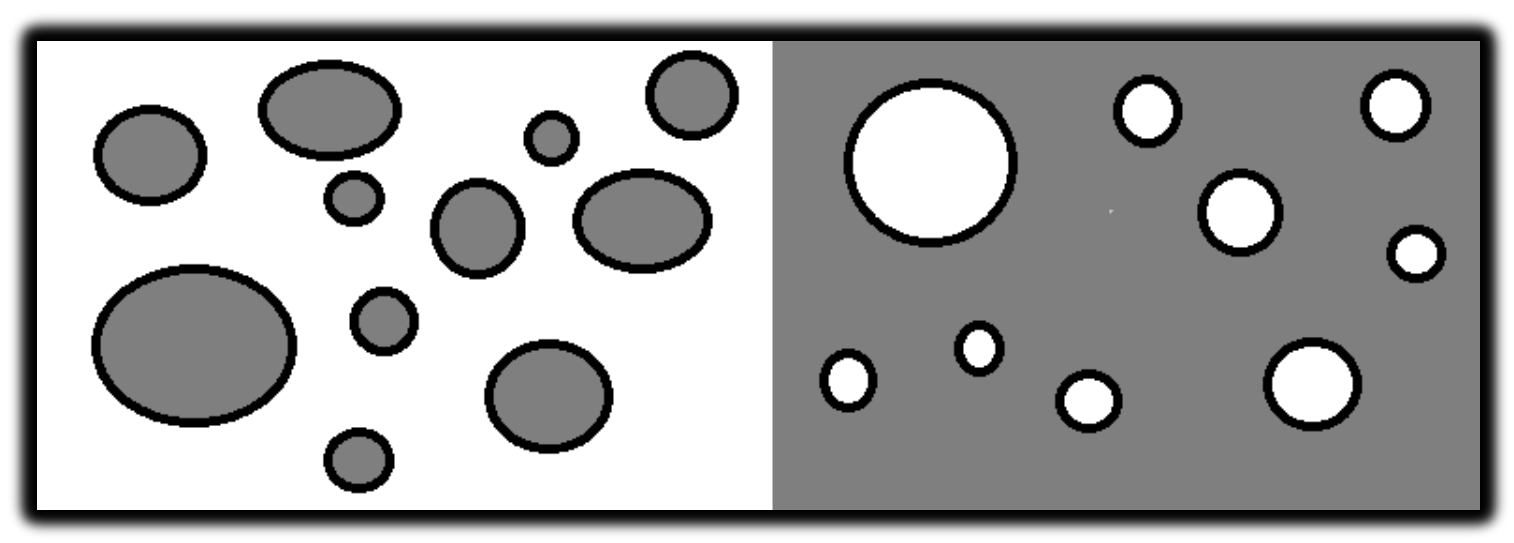

Keywords: supercritical water, hydrogen bonding, density inhomogeneities, molecular dynamics simulation, structural transitions

\section{Highlights}

- The structural properties of supercritical water along a near-critical isotherm have been studied.

- Classical molecular dynamics simulation techniques have been employed.

- Hydrogen bonding interactions play a key role in the formation of local density inhomogeneities.

- There is significant evidence about the existence of a liquid-like to gas-like structural transition in supercritical water. 


\section{Introduction}

Supercritical water (SCW) is an environmental friendly solvent and a cheap alternative to organic ones, having thus a wide applicability in several green chemical processes [1-5]. The main reason for this wide applicability is that by slightly adjusting the thermodynamic conditions, the dielectric properties of SCW can be drastically altered, allowing thus the eclectic dissolution of several types of polar or non-polar solutes in SCW. Such a behavior, in combination with the low viscosity of $\mathrm{SCW}$, has promoted its use in new emerging applications such as hazardous waste treatment, biomass processing and materials synthesis. Apart from the technological applications, the study of the properties of SCW is also very important in Geochemistry. SCW acts as a medium in several hydrothermal reactions in the Earth's crust and mantle [6] and can also be dissolved in silicate minerals, strongly affecting in this way many important geochemical processes.

The properties of SCW exhibit in general significant differences with its liquid state ones [7-20]. According to several existing experimental and simulation data [21-41] the hydrogen bonding ( $\mathrm{HB}$ ) network in SCW is much weaker and the fraction of water molecules forming hydrogen bonds is much lower at supercritical conditions. However, water molecules can still form hydrogen bonds even at these high temperature conditions and quite recent experimental studies have confirmed the existence of hydrogen bonds up to at least $873 \mathrm{~K}$ and $132 \mathrm{MPa}$ [20]. Previous studies have also confirmed the existence of local density inhomogeneities [29, 42] in SCW and have revealed that the local density augmentation is much more pronounced in comparison with non-HB fluids [29, 42-44]. Taking into account the importance of density inhomogeneities in determining a wide range of properties of supercritical fluids [45]' a deeper insight on the interplay between hydrogen bonding and the local structural fluctuations in SCW would lead towards a better understanding of its behavior.

\footnotetext{
${ }^{1}$ Abbreviations:

SCW: Supercritical Water, HB: Hydrogen Bonding, MD: Molecular Dynamics, rdf: radial distribution function, LDA: Local Density Augmentation
} 
Molecular simulation has become a very important tool to investigate the properties of supercritical fluids and their solutions, as pointed out in recent reviews [46]. The present study has been therefore devoted to a more thorough investigation of this interplay by performing a series of molecular dynamics simulations of SCW along a near critical isotherm, aiming to shed light on open questions regarding the interrelation between hydrogen bonding, local density inhomogeneities and structural order in SCW. Moreover, attention has been paid to provide information related to recent observations [41], according to which at near-critical temperatures there might be a supercritical fluid - gas transition at densities lower than $0.2 \rho_{c}$, due to the collapse of the short-range order. This work aims therefore to provide additional insight towards a deeper understanding of a wide-range of properties of SCW, often related to it peculiar behavior near the critical point.

The computational details of the performed simulations are presented in Section 2 of the manuscript. The results obtained and the following discussion is presented in Section 3. Finally, Section 4 contains the general conclusions and remarks drawn from the present study.

\section{Computational Details}

Eleven thermodynamic state points of SCW were simulated in the framework of the present study. Ten molecular dynamics (MD) atomistic simulations of SCW were performed at a constant near-critical temperature of $\mathrm{T}=666 \mathrm{~K}\left(\mathrm{~T}_{\mathrm{c}}=647.1 \mathrm{~K}\right)$ and for densities in the range $0.0644-0.644 \mathrm{~g} / \mathrm{cm}^{3}\left(\rho_{c}=0.322 \mathrm{~g} / \mathrm{cm}^{3}\right)$ in order to reveal the effect of hydrogen bonding interactions on the local structural order and density inhomogeneities in SCW. An additional MD simulation was later performed at the lower density of $0.0322 \mathrm{~g} / \mathrm{cm}^{3}$ and at the same temperature, in order to investigate the possible existence of structural transitions in SCW. The simulated state points of SCW are presented in Table I. The simulations were carried out with 500 molecules, using the DL_POLY software [47]. Each simulation was extended to $1 \mathrm{~ns}$ to achieve equilibrium, starting from an initial configuration created with the packmol software [48], and the properties of SCW were evaluated in subsequent simulations with duration of 2 ns. In all simulations the equations of motion were integrated using a leapfrog-type Verlet algorithm and the integration time step was set to $1 \mathrm{fs}$. The Nose- 
Hoover thermostat [49] with a temperature relaxation time of 0.5 ps was also used to constrain the temperature during the simulations. The intramolecular geometry of the molecules was also constrained by employing the shake algorithm [50].

The rigid SPC/E potential model [51] was employed to describe the site-site interactions between the water molecules. This three-site potential model has been successfully used in previous studies of liquid water and SCW, as well as in the case of several aqueous mixtures. Therefore, our choice has been based upon previously reported simulation results concerning the accuracy of this model in approximating sufficiently the critical point of water [35,36], as well as various properties of the fluid $[35,51-53]$. Taking into account that the performance of $\mathrm{SPC} / \mathrm{E}$ in predicting the properties of SCW is very reasonable, as well as that our previous studies on SCW have also been performed with the same model [29,30,42,52,54], we decided to employ it in the present treatment as well. In such a way our findings could be also correlated to the findings of our previous works, providing in this way a more complete picture of the behavior of SCW at near-critical, supercritical conditions. It has to be noted that in the presentation of the results in this study, the simulated fixed densities have been presented as reduced densities $\rho / \rho_{c}$, taking the experimental critical density as a reference. The reason to do so is that the reported values for the calculated critical density using the SPC/E model are close to the experimental one (for instance $0.29 \mathrm{~g} / \mathrm{cm}^{3}$ in reference 35 and $0.308 \mathrm{~g} / \mathrm{cm}^{3}$ in reference 36). Moreover, since depending on the method used to extract the value of the critical density in the simulations the calculated values can be slightly different (as in references 35, 36), it was considered as a more standard approach to use the experimental critical density as a reference point. The temperature of the simulated isotherm in our case is also higher than the predicted critical temperatures of SPC/E water $[35,36]$, ensuring that the simulated fluid is at supercritical conditions [45]. In our simulations a cut-off radius of 12.0 $\AA$ has been applied for LJ interactions and long-range corrections have been also taken into account. To account for the long-range electrostatic interactions the Ewald summation technique was used. 


\section{Results and Discussion}

\section{A. HB effect on local structure and density augmentation}

In order to obtain a clearer picture of the effect of $\mathrm{HB}$ interactions on the local structural properties of SCW the individual water molecules in the fluid were classified in different types according to the number of hydrogen bonds they form with other water molecules. Note that there are several definitions of the hydrogen bond in the literature [28], but a well-established geometric criterion, also used in several previous studies [25-27, 29, 30], was used in order to analyze the HB network in SCW. According to this criterion a hydrogen bond between two water molecules exists if the interatomic distances are such that RO..O $\leq 3.6 \AA$, RH... $\leq 2.4 \AA$ and the donor-acceptor angle $\mathrm{H}-\mathrm{O} \ldots \mathrm{O} \leq 30^{\circ}$ (the symbol ... corresponds to intermolecular distances). Since our previous studies [29] have revealed that the main trends of the density dependence of the number of hydrogen bonds in several supercritical fluids and corresponding statistics can be realistically represented by simple geometric criteria and since the systematic study of HB definitions is way out of the scope of the present treatment, this simple definition was employed in the present study. Based on this criterion the fractions of water molecules forming 0-4 hydrogen bonds were estimated for all the investigated state points and are present as a function of the bulk density of the fluid in Figure 1. From this figure it can be clearly seen that in the range of the simulated bulk densities the majority of water molecules from mainly 0-2 hydrogen bonds and there is also a small fraction of water molecules forming 3 hydrogen bonds, which increases with the density. The fraction of water molecules forming 4 hydrogen bonds is almost negligible. It is interesting to notice that the fraction of water molecules forming one hydrogen bond maximizes in the density range $0.8-1.0 \rho_{c}$ and then starts to decrease. The fractions of molecules forming two hydrogen bonds increases non-linearly with the density and the fraction of HB-free molecules decreases also non-linearly as the density increases.

Our previous studies on SCW [29] have revealed that the mean number of hydrogen bonds per water molecule exhibits a clearly non-linear bulk density dependence, which is much more pronounced in SCW in comparison with other HB supercritical fluids like methanol, ethanol and ammonia. This behavior has been related as one of the main reasons for the observation of strong local density augmentation (LDA) 
effects in SCW and for the observation of a maximum in the local density augmentation, which was located at $0.68 \rho_{c}$. From the present analysis it can be observed that the fractions of molecules forming one and two hydrogen bonds exhibit similar bulk density dependence, signifying that they have the most important contribution on the bulk density dependence of the average LDA.

The determination of the LDA in supercritical fluids is based upon the calculation of the pair radial distribution functions (rdf) in order to calculate the corresponding local coordination numbers and effective local densities. The use of effective local densities to investigate local density inhomogeneities in supercritical fluids has been extensively discussed and justified in previous publications [42-44, 55-57]. To calculate the effective local densities in SCW, we initially calculated the average coordination number $\mathrm{N}_{\mathrm{c}}$ corresponding to the first solvation shell of SCW at each state point:

$$
\mathrm{N}_{c}\left(\rho, R_{c}\right)=4 \pi \rho \int_{0}^{R_{c}} g_{o O}(r) r^{2} d r
$$

In this equation, $g_{o o}(r)$ is the oxygen-oxygen $\mathrm{rdf}$, which is essentially identical to the center of mass (com) - center of mass one, and $\rho$ is the bulk number density of the fluid. $R_{c}$ is a defined cut-off distance specifying the solvation shell around a water molecule. To estimate the excess local density in a supercritical fluid, it is more convenient to calculate the effective local density [42-44, 55-57], $\rho_{\text {eff }, l}$, according to the following relation

$$
\rho_{\text {eff }, l}\left(\rho, R_{c}\right)=\frac{\mathrm{N}_{c}\left(\rho, R_{c}\right)}{\mathrm{N}_{c}\left(\rho_{r e f}, R_{c}\right)} \rho_{r e f}
$$

The employed reference density, $\rho_{\text {ref }}$, corresponds typically to a high liquid-like density and has been set as $2 \rho_{c}$. The cut-off distance $R_{c}$ is determined as the position of the first minimum of the corresponding $g_{o O}(r)$ observed at this high reference density $\rho_{r e f}$. In the case of SCW the estimated value of $R_{c}$ is $4.22 \AA$. This cut-off distance has been used to compute the effective local density, $\rho_{\text {eff }, l}$, of the first 
coordination shell in all the simulated state points of interest. The LDA has been therefore defined as $\Delta \rho_{\text {eff }, l}=\rho_{\text {eff }, l}-\rho$.

In order to find the interrelation between HB interactions and the LDA in SCW, we initially focused on the effect of the $\mathrm{HB}$ state of the water molecules on the determination of the rdfs around them. The term HB state in this case is associated to the number of hydrogen bonds formed by each individual water molecule. Since in the case of water the O-O rdf is essentially identical to the com-com rdf, we focused only on this particular rdf. The bulk density dependence of the O-O rdf for water molecules forming 0-3 hydrogen bonds is presented in Figures 2,3. It is interesting to note that a weak first peak located at $3 \AA$ is observed for HB-free molecules, followed by a second, higher-intensity peak at $3.4 \AA$. This first low-intensity peak, which turns to a lower intensity shoulder at higher bulk densities, is an indication of the weak short-range interactions of non-HB water dimers located at short intermolecular distances. These weakly interacting dimers are quite scarce and the probability to find most of the non-HB water dimers at longer intermolecular distances is higher. That's why the main peak is located at $3.4 \AA$ in the case of non-HB water molecules. On the other hand, in the case of hydrogen bonded molecules there is just one strong peak located at $3 \AA$, due to the existence of HB interactions. The intensity of this peak increases with the number of hydrogen bonds formed by the central water molecule. Regarding the bulk density dependence of these functions, the intensity of the peaks decreases as the bulk density increases in all cases. Such a finding is in agreement with previous results in the literature.

From these calculated rdfs the composition of the solvation shell of water molecules can be predicted by calculating the corresponding coordination numbers for all classes of water molecules. The cut-off used in determining the size of the solvation shell was $4.22 \AA$ in all cases. The dependence of the coordination number on the bulk density is presented in Figure 4. From this figure it can be clearly observed that the deviations from linearity are more pronounced when the number of hydrogen bonds formed by the reference water molecules increases. This is a clear indication of the strong HB-effect on the formation of LDA in SCW. Also, as the bulk density increases the differences between the calculated coordination numbers for the different classes of molecules decrease. For example, the calculated coordination 
numbers for molecules forming zero and three hydrogen bonds at $0.2 \rho_{c}$ are 0.83 and 3.89, respectively. The corresponding coordination numbers at $2 \rho_{c}$ are 5.92 and 6.94. This behavior is related to the more dense packing of SCW at higher densities, which at densities close to $2 \rho_{c}$ starts to approach a liquid-like behavior and therefore the local density inhomogeneities are much less pronounced.

The average coordination number can be expressed as a linear combination of these coordination numbers when the fractions of molecules forming 0-3 hydrogen bonds are known. Therefore it can be expressed as: $N_{c}=\sum_{i} \chi_{i} \cdot N_{c}^{i}$. In this equation, $\chi_{i}$ is the molar fraction of molecules forming $\mathrm{i}=0-3$ hydrogen bonds and $N_{c}^{i}$ is the coordination number corresponding to each class of molecules. The contributions $\chi_{i} \cdot N_{c}^{i}$ to the total average coordination number for the classes of molecules forming $\mathrm{i}=0-3$ hydrogen bonds for all the investigated bulk densities are also presented in Figure 4. From these data it can be seen that the most important contribution to the overall average coordination number arises from the fraction of molecules forming mainly one and two hydrogen bonds. The contribution of HB-free molecules is also important in the range 0.2-1.0 $\rho_{c}$ and then it starts to decrease. On the other hand, the contribution of molecules forming three hydrogen bonds starts to become more important for densities higher than the critical one where SCW begins to approach a liquid-like behavior.

In order to estimate the contribution of these different classes of molecules to the overall average LDA, it was expressed in the form:

$$
\Delta \rho_{\text {eff }, l}=\rho_{\text {eff }, l}-\rho_{\text {bulk }}=\frac{\mathrm{N}_{c}\left(\rho, R_{c}\right)}{\mathrm{N}_{c}\left(\rho_{\text {ref }}, R_{c}\right)} \rho_{\text {ref }}-\rho_{\text {bulk }}=\frac{\sum_{i} \chi_{i} \cdot N_{c}^{i}}{N_{r e f}} \rho_{r e f}-\rho_{\text {bulk }}
$$

The above equation can be further expressed in the following way:

$$
\Delta \rho_{e f f, l}=\sum_{i}\left(\chi_{i} \cdot N_{c}^{i} \cdot \frac{\rho_{r e f}}{N_{r e f}}\right)-\sum_{i}\left(\chi_{i} \cdot \rho_{b u l k}\right)
$$

which at the end gives the final expression: 


$$
\Delta \rho_{e f f, l}=\sum_{i}\left(\chi_{i} \cdot N_{c}^{i} \cdot \frac{\rho_{\text {ref }}}{N_{\text {ref }}}-\chi_{i} \cdot \rho_{\text {bulk }}\right)=\sum_{i}\left\{\chi_{i} \cdot\left(\frac{N_{c}^{i}}{N_{\text {ref }}} \cdot \rho_{\text {ref }}-\rho_{\text {bulk }}\right)\right\}
$$

Using equation 5 , the contributions of each class of water molecules to the overall average LDA has been estimated and it is plotted as a function of the bulk density in Figure 5. From this figure it may be clearly observed that the contribution of non-HB molecules is very small, and specifically for bulk densities higher than $0.6 \rho_{c}$ it is negative. This is a clear evidence of the important role of HB interactions on the more pronounced LDA effects in SCW in comparison with non-HB fluids [29]. On the other hand, the most important contributions to the average LDA come from molecules mainly forming one and two hydrogens bonds and to a much lesser extent from molecules forming three hydrogen bonds. The contributions of molecules forming one hydrogen bond are more important in the bulk density range $0.2-1.0 \rho_{c}$, whereas the contribution of molecules forming two hydrogen bonds is more important in the range $1.0-2.0 \rho_{c}$.

\section{B. Local Structural Order}

Apart from the description of the structure in SCW in terms of rdfs and coordination numbers, in the present study particular attention was given to the structural order around the water molecules. In general, this structural order can be described in terms of orientational order parameters. The most commonly used order parameter in the case of water is the tetrahedral order parameter $q_{4}[58,59]$. This parameter gives information about the extent to which a molecule and its four nearest neighbors adopt a tetrahedral arrangement and is defined as:

$$
q_{4}=1-\left\langle\frac{3}{8} \sum_{j=1}^{3} \sum_{k=j+1}^{4}\left(\cos \phi_{j i k}+\frac{1}{3}\right)^{2}\right\rangle
$$

In this equation $\phi_{j i k}$ corresponds to the angle formed by the vectors $\vec{r}_{i j}$ and $\vec{r}_{i k}$, connecting the oxygen atom of the central molecule $i$ with the oxygen atoms of two of its four nearest neighbors $\mathrm{j}$, k. Using this definition the parameter $q_{4}$ gets the value $\mathrm{q}=1$ in a perfect tetrahedral network and the value $q_{4}=0$ in an ideal gas [58]. 
Previous studies in the literature $[41,60]$ have revealed that $q_{4}$ in SCW shows significant density dependence and it is generally low, even at liquid-like densities. In the present treatment the existence of different kinds of local structures around the water molecules was also studied, since our previous studies have revealed that $q_{4}$ is strongly related to the formation of four hydrogen bonds around the water molecules. Taking also into account that only up to three hydrogen bonds can be formed by the water molecules in the investigated range of thermodynamic conditions, the trigonal order parameter $q_{3}[61,62]$ of SCW was investigated for the first in the framework of the present study. The $q_{3}$ order parameter is defined, taking into account the three closest neighbors of a given molecule $\mathrm{i}$, as:

$$
q_{3}=1-\left\langle\frac{4}{7} \sum_{j=1}^{2} \sum_{k=j+1}^{3}\left(\cos \phi_{j i k}+\frac{1}{2}\right)^{2}\right\rangle
$$

As in the case of the tetrahedral order parameter, this order parameter has been normalized to one for perfect order and zero for a random distribution.

The dynamic behavior of these parameters has also been investigated, by calculating the corresponding average time correlation functions [63]:

$$
C_{q_{n}}(t)=\frac{\left\langle\delta q_{n}(0) \cdot \delta q_{n}(t)\right\rangle}{\left\langle\delta q_{n}(0)^{2}\right\rangle}, \delta q_{n}(t)=q_{n}(t)-\left\langle q_{n}\right\rangle
$$

The corresponding correlation times $\tau_{q_{n}}$ associated with the relaxation of the corresponding orientational order parameter have been calculated using the relation:

$$
\tau_{q_{n}}=\int_{0}^{\infty} C_{q_{n}}(t) \cdot d t
$$

The bulk density dependence of $q_{3}$ and $q_{4}$, as well as of their corresponding relaxation times $\tau_{q_{3}}$ and $\tau_{q_{4}}$, are plotted in Figure 6. From this figure it can be clearly seen that the values of $q_{3}$ are higher than the $q_{4}$ ones at all the investigated thermodynamic state points and they all increase non-linearly with the bulk density. 
This is a clear indication that the structural orientational order is also strongly related to $\mathrm{HB}$ interactions and LDA in SCW. The bulk density dependence of the corresponding relaxation times $\tau_{q_{3}}$ and $\tau_{q_{4}}$ on the other hand has a very similar behaviour with local density dynamics of supercritical fluids at more extended length scales, beyond the first or second solvation shell, as it has been observed in our previous studies [29]. These relaxation times increase up to about 0.6-0.8 $\rho_{c}$ and then they decrease rapidly with the increase of the bulk density. The fact that the density dependence of the structural order parameters' and local density dynamics is very similar is probably related to the fact that the local density dynamics slowing-down in the density range $0.6-0.8 \rho_{c}$ causes a corresponding slowing-down of the overall packing dynamics of water molecules. And this slowing-down is also reflected on the dynamics of the structural order changes, which are directly related to the packing of the water molecules around a central one. The values of $\tau_{q_{4}}$ are also slightly higher than the corresponding $\tau_{q_{3}}$ ones. This could be explained if someone takes into account that the region around a molecule containing its four nearest neighbors is more extended in comparison with the region containing its three nearest neighbors. Previous studies [29,45] have revealed that the local density relaxation times of supercritical fluids increase with the increase of the size of the region taken into account in the calculation. In this sense, this finding is in agreement with all these previously reported trends.

Taking into account that by definition the tetrahedral and trigonal order parameters depend upon the distribution of nearest neighbors, this signifies that the closest three and four neighbors of each individual water molecule are distributed over a more extended length scale of the first and second solvation shell, which are located at 4.22 and $6.75 \AA$, respectively at the bulk density of $2.0 \rho_{c}$.

In order to provide a deeper description of these effects, the distribution of nearest neighbors and their contribution on the overall shape of the O-O rdfs obtained were systematically explored by employing a methodology similar to the one used in recent studies $[64,65]$. The nearest neighbors have been classified by sorting their distances from a central molecule. According to the methodology used, the overall site-site rdf 
can be also analyzed as a sum of the rdfs $g_{a-b}{ }^{n}(r)$, each one corresponding to the $\mathrm{n}^{\text {th }}$ neighbor molecule around a central one:

$$
g_{a-b}(r)=\sum_{n=1}^{\infty} g_{a-b}^{n}(r)
$$

The contributions up to the fourth nearest neighbors to the overall $\mathrm{O}-\mathrm{O}$ rdfs for representative investigated state points is presented by means of the functions $g^{n}(r)$, where $n=1-4$, in Figures 7-8. From these figures it can be observed that the distribution of the third and fourth neighbor can be extended to larger length scales than the size of the first solvation shell, or even the second solvation shell at low bulk densities, and they are much broader in comparison with the corresponding distributions of the first and second neighbor. All these distributions are also less symmetric at low bulk densities, especially the ones corresponding to the first and second neighbor, starting to exhibit a more symmetric shape at bulk densities higher than $\rho_{c}$. In the case of the first neighbor the contribution of $g^{1}(r)$ to the short-range shape of the total $g(r)$ is the most important at lower densities and its intensity decreases with the bulk density increase. However, according to our results, the contribution of $g^{2}(r)$ seems to be more important in the density range $0.4-0.8 \rho_{c}$ and then its intensity decreases. On the other hand, the peak intensity of $g^{3}(r)$ and $g^{4}(r)$ is higher at high densities and decreases with the decrease of the bulk density. These findings are consistent with the bulk density dependence of the calculated order parameters $q_{3}$ and $q_{4}$ and their corresponding relaxation times. The maximization of these relaxation times in the density range $0.6-0.8 \rho_{c}$ is also consistent with the fact that the LDA is maximized in the same range, slowing down in this way the local density and the structural order relaxation dynamics.

Finally, the dependence of the calculated order parameters of the individual water molecules upon the number of hydrogen bonds they form was also investigated. The bulk density dependence of $q_{3}$ and $q_{4}$ for water molecules forming 0-3 hydrogen bonds is presented in Figure 9. From this figure it can be observed that there is a very strong coupling among HB interactions and these local orientational order parameters. As the degree of $\mathrm{HB}$ increases, the values of $q_{3}$ and $q_{4}$ increase significantly. 
Moreover, the bulk density dependence of these order parameters is weaker for more strongly hydrogen bonded water molecules, especially the ones forming 3 hydrogen bonds. From Figure 9 it can be seen that the $q_{3}$ values corresponding to molecules forming 3 hydrogen bonds remain almost constant at all bulk densities, signifying that HB interactions are the most crucial factor in determining the local orientational order around the water molecules. Similar conclusions were drawn in our previous ab initio molecular dynamics studies of liquid water [62, 63], signifying that HB interactions have a very important role in determining the local orientational structural order around water molecules in a very wide range of thermodynamic conditions.

\section{Evidence of Structural Transitions}

Interestingly, while inspecting the relevant literature, we noticed that in a previous simulation study of SCW along the same isotherm, the authors reported evidences of a structural transition taking place at the density of about $0.19 \rho_{c}$. In that study [41] the authors calculated the mean relative fluctuation $\sqrt{(\Delta E)^{2}} /<E>$ of the water-water interaction energy within the first solvation shell and they found out that it increases sharply at $0.19 \rho_{c}$. They attributed this behavior to a possible supercritical fluid - gas structural transition taking place at these conditions, where the water molecules tend to locate arbitrarily around a center water molecule due to greatly enhanced fluctuation regardless of its favorable or non-favorable energy.

At the same period when the above mentioned study was published, another interesting independent study published by Sedunov [66], similarly suggested a fluid structural transition in supercritical fluids, going from a liquid-like structure to a gaslike one. The conclusions of that study were based on the interpretation of available thermodynamic experimental data. According to Sedunov [66], the liquid-like structure resembles a continuous condensed medium filled with different size pores and small density islands. On the other hand, the gas-like structure resembles a continuous vacuum medium filled with clusters of different dimensions and with a variety of types of intermolecular bonding. A schematic representation of the liquidlike and gas-like structures is provided in Figure 10. 
In order to provide a more structure-based analysis of these phenomena under investigation, apart from the $0.2-2.0 \rho_{c}$ investigated range of densities we performed an additional simulation at $0.1 \rho_{c}$ and applied a new methodology to search for possible structural transitions in this particular range of thermodynamic conditions. As it has been mentioned above, the tetrahedral order is one of the most characteristic features of the structure of liquid water and our present study revealed interesting findings regarding its interconnection with local density inhomogeneities in SCW. This order parameter is related to the orientational ordering of the four nearest neighbors around a central water molecule. However, it does not provide information about the length scale of the region where these neighbors are located, which could provide an additional measure of the resemblance of SCW with a liquidlike fluid. Our decomposition of the O-O rdfs in terms of the contributions of nearest $\mathrm{n}$ neighbors, as reflected in the shape of the calculated functions $g^{n}(r)$, could provide valuable information about the location of these nearest neighbors. By carefully inspecting Figure 8, it might be observed that the shape of the $g^{3}(r), g^{4}(r)$ functions starts to change significantly when approaching the density of $0.2 \rho_{c}$. At this density it can be observed that these distributions become much broader and extend up to a significantly larger length scale. In order to provide a description of this length scale and to find possible changes when going from $0.2 \rho_{c}$ to $0.1 \rho_{c}$, we calculated the average volume of the sphere around a central water molecule which contains its four nearest neighbors. The radius of this sphere is determined by the average oxygen-oxygen distance between each individual water molecule and its fourth nearest neighbor.

The calculated average volume $\mathrm{V}_{4}$ of this sphere is presented in Figure 11. From this figure a very sharp increase in the volume $\mathrm{V}_{4}$ is observed when going from $0.2 \rho_{c}$ to $0.1 \rho_{c}$. Such a finding is a indication that in this range of densities the short-range order is destroyed and the liquid-like to gas-like fluid structural transition takes place, further supporting the previously reported works $[41,66]$.

The relaxation dynamics of this volume were also studied by calculating the corresponding time correlation functions and relaxation times: 


$$
C_{V_{4}}(t)=\frac{\left\langle\delta V_{4}(0) \cdot \delta V_{4}(t)\right\rangle}{\left\langle\delta V_{4}(0)^{2}\right\rangle}, \quad \delta V_{4}(t)=V_{4}(t)-\left\langle V_{4}\right\rangle, \quad \tau_{V_{4}}=\int_{0}^{\infty} C_{V_{4}}(t) \cdot d t
$$

The calculated correlation times $\tau_{V_{4}}$, associated with the relaxation of the volume of the sphere containing the closest four neighbors of each individual water molecule, as a function of the bulk density of the system are also presented in Figure 11. From this figure it can be clearly observed that these correlation times decrease with the increase of the density, exhibiting a sigmoidal-like decay with the increase of the density. At the density range where the structural transition takes place, the correlation time remains almost constant, signifying that in this range of densities the system exhibits a gas-like behavior. On the other hand, in the liquid-like supercritical region (densities higher than $0.2 \rho_{c}$ ) the relaxation dynamics are very much affected by the local environment around the water molecules and as the density increases the corresponding volume fluctuation dynamics become significantly faster.

As it has been pointed out in Ref. 66, the fluid structural transition is not really a phase transition (either first- or second-order), because both the gas and liquid phases coexist in the fluid in forms of clusters and pores. During these structural transitions, significant but not abrupt changes of thermophysical properties can be observed. However, other previous studies also revealed that there are some thermophysical quantities exhibiting maxima, which define a line emanating from the critical point, named as the Widom line in the case of the constant-pressure specific heat [67]. These previous studies support that this line actually divides the supercritical region into two regions which, although not connected by a first-order singularity, can be identified by different dynamical regimes: gas-like and liquid-like, reminiscent of the subcritical domains. Our study directly attributes the existence of the Widom line to the structural transitions in SCW. And the simple geometrical method proposed in our study could provide an alternative way to locate the Widom line, not based upon the behavior of thermodynamic properties but directly upon some specific structural features of the fluid. However, extensive studies in a wide range of thermodynamic conditions are required in order to verify this and it is one of our main goals for the near future. Research on this field is in progress. 


\section{Conclusions}

Classical molecular dynamic simulation techniques were employed in the present treatment to investigate the interplay among hydrogen bonding, local structural order and density inhomogeneities in supercritical water, along a near-critical isotherm. The results obtained have revealed the important role of $\mathrm{HB}$ interactions on the more pronounced LDA effects in SCW in comparison with non-HB fluids. Especially water molecules forming one and two hydrogen bonds have a very strong effect on the determination of the local density augmentation in the fluid. Particular attention was also given to the structural orientational order around the water molecules and its relation to $\mathrm{HB}$ interactions, by calculating the trigonal and tetrahedral order parameters. The results obtained revealed that the structural orientational order in $\mathrm{SCW}$ is also strongly related to HB interactions and LDA. Interestingly, the relaxation times of these order parameters are maximized in the density range 0.6-0.8 $\rho_{c}$, something which is also consistent with the fact that the LDA is maximized in the same range, slowing down in this way the local density and the structural order relaxation dynamics. The structural analysis based upon the identification of nearest neighbors and particularly the estimation of the volume $\mathrm{V}_{4}$ of the sphere containing the four nearest neighbors around a central water molecule revealed a very sharp increase of this volume when going from $0.2 \rho_{c}$ to $0.1 \rho_{c}$. Such a finding provides an indication that in this range of densities the short-range order is destroyed and a liquid-like to gas-like fluid structural transition takes place in the system.

\section{Acknowledgements}

I.S. acknowledges the financial support from the group of Professor Elvira Guardia (Computer Simulation in Condensed Matter Research Group - SIMCON), which allowed him to visit the Department of Physics of the Technical University of Catalonia (UPC) for one month, in order to work on the structure and dynamics of water at several different thermodynamic conditions. The use of the computational facilities of the SIMCON group and of the Physical Chemistry Laboratory at the Department of Chemistry - University of Athens is also gratefully acknowledged. E.G. acknowledges financial support from the Ministerio de Economía y Competitividad of Spain, Grant FIS2015-66879-C2-1-P (MINECO/FEDER). 


\section{References}

1) H. Weingärtner, E.U. Franck, Supercritical water as a solvent, Angew. Chem. Int. Ed 44 (2005), 2672-2692

2) E. Kiran, P.G. Debenedetti, C.J. Peters (Eds.), Supercritical Fluids: Fundamentals and Applications, NATO ASI Science Series E, Applied Sciences, Kluwer Academic Publishers, Dordrecht, 2000, Vol. 366.

3) A. Kruse, E. Dinjus, Hot compressed water as reaction medium and reactant: Properties and synthesis reactions, J. Supercrit. Fluids 39 (2007), 362-380.

4) N. Akiya, Ph. Savage, Roles of water for chemical reactions in high-temperature water. Chem. Rev. 102 (2002), 2725-2750.

5) G. Galli, D. Pan, A closer look at supercritical water, Proc. Natl. Acad. Sci. U.S.A. 110 (2013), 6250-6251.

6) M. Hirschmann, D. Kohlstedt, Water in Earth's mantle, Phys. Today 65 (2012), $40-45$.

7) P. Postorino, R. H. Tromp, M. A. Ricci, A. K. Soper, G. W. Neilson, The interatomic structure of water at supercritical temperatures, Nature 366 (1993), 668670.

8) Y. E. Gorbaty, A. G. Kalinichev, Hydrogen Bonding in Supercritical Water. 1. Experimental Results. J. Phys. Chem. 99 (1995), 5336-5340.

9) M. C. Bellissent-Funel, T. Tassaing, H. Zhao, D. Beysens, B. Guillot, Y. Guissani, The structure of supercritical heavy water as studied by neutron diffraction, J. Chem. Phys. 107 (1997), 2942-2949.

10) M. M. Hoffmann, M. S. Conradi, Are There Hydrogen Bonds in Supercritical Water?, J. Am. Chem. Soc. 119 (1997), 3811-3817.

11) A. K. Soper, F. Bruni, M. A. Ricci, Site-site pair correlation functions of water from 25 to $400{ }^{\circ} \mathrm{C}$ : Revised analysis of new and old diffraction data, J. Chem. Phys., 106 (1997), 247-254. 
12) A. Botti, F. Bruni, M. A. Ricci, A. K. Soper, Neutron diffraction study of high density supercritical water, J. Chem. Phys. 109 (1998), 3180-3184.

13) A. K. Soper, The radial distribution functions of water and ice from 220 to $673 \mathrm{~K}$ and at pressures up to $400 \mathrm{MPa}$, Chem. Phys. 258 (2000), 121-137.

14) R. H. Tromp, P. Postorino, G. W. Neilson, M. A. Ricci, A. K. Soper, Neutron diffraction studies of $\mathrm{H}_{2} \mathrm{O} / \mathrm{D}_{2} \mathrm{O}$ at supercritical temperatures. A direct determination of $\mathrm{g}_{\mathrm{Hн}}(\mathrm{r}), \mathrm{g}_{\mathrm{OH}}(\mathrm{r})$ and $\mathrm{g}_{\mathrm{OO}}(\mathrm{r})$, J. Chem. Phys. 101 (1994), 6210-6215.

15) T. Tassaing, M. C. Bellissent-Funel, B. Guillot, Y. Guissani, The partial pair correlation functions of dense supercritical water, Europhys. Lett. 42 (1998), 265270.

16) N. Matubayasi, C. Wakai, M. Nakahara, NMR Study of Water Structure in Superand Subcritical Conditions, Phys. Rev. Lett. 78 (1997), 2573-2576.

17) Ph. Wernet, D. Testernale, J. L. Hazemann, R. Argoud, P. Glatzel, L. G. M. Pettersson, A. Nilsson, U. Bergmann, Spectroscopic characterization of microscopic hydrogen-bonding disparities in supercritical water, J. Chem. Phys. 123 (2005), 154503.

18) A. K. Soper, Bridge over troubled water: the apparent discrepancy between simulated and experimental non-ambient water structure, J. Phys. : Condens. Matter 8 (1996), 9263-9267.

19) Q. Sun, Q. Wang, D. Ding, Hydrogen Bonded Networks in Supercritical Water, J. Phys. Chem. B 118 (2014), 11253-11258.

20) C. J. Sahle, C. Sternemann, C. Schmidt, S. Lehtola, S. Jahn, L. Simonelli, S. Huotari, M. Hakala, T. Pylkkänen, A. Nyrow, K. Mende, M. Tolan, K. Hämäläinen, M. Wilke, Microscopic structure of water at elevated pressures and temperatures, Proc. Natl. Acad. Sci. U.S.A. 110 (2013), 6301-6306.

21) P. Jedlovszky, J. B. Brodholt, F. Bruni, M.A. Ricci, A. K. Soper, R. Vallauri, Analysis of the hydrogen-bonded structure of water from ambient to supercritical conditions, J. Chem. Phys. 108 (1998), 8528-8540. 
22) A. G. Kalinichev, S. V. Churakov, Thermodynamics and structure of molecular clusters in supercritical water, Fluid Phase Equil. 183 (2001), 271-278.

23) A. G. Kalinichev, S. V. Churakov, Size and topology of molecular clusters in supercritical water: a molecular dynamics simulation, Chem. Phys. Lett. 302 (1999), 411-417.

24) T. I. Mizan, P. E. Savage, R. M. Ziff, Temperature Dependence of Hydrogen Bonding in Supercritical Water, J. Phys. Chem. 100 (1996), 403-408.

25) C. Nieto-Draghi, J. Bonet Avalos, B. Rousseau, Dynamic and structural behavior of different rigid nonpolarizable models of water, J. Chem. Phys. 118 (2003), 79547964.

26) J. Marti, Dynamic properties of hydrogen-bonded networks in supercritical water, Phys. Rev. E. 61 (2000), 449-456.

27) J. Marti, Analysis of the hydrogen bonding and vibrational spectra of supercritical model water by molecular dynamics simulations, J. Chem. Phys. 110 (1999), 68766886.

28) A. G. Kalinichev, J. D. Bass, Hydrogen Bonding in Supercritical Water. 2. Computer Simulations, J. Phys. Chem. 101 (1997), 9720-9727.

29) I. Skarmoutsos, D. Dellis, J. Samios, The Effect of Intermolecular Interactions on Local Density Inhomogeneities and Related Dynamics in Pure Supercritical Fluids. A Comparative Molecular Dynamics Simulation Study, J. Phys. Chem. B 113 (2009), $2783-2793$.

30) I. Skarmoutsos, E. Guardia, Effect of the local hydrogen bonding network on the reorientational and translational dynamics in supercritical water, J. Chem. Phys. 132 (2010), 074502.

31) G. Löfler, H. Schreiber, O. Steinhauser, Computer Simulation as a Tool to Analyze Neutron Scattering Experiments: Water at Supercritical Temperatures, Ber. Bunsenges. Phys. Chem. 98 (1994), 1575-1578.

32) E. S. Fois, M. Sprik, M. Parinello, Properties of supercritical water: an ab initio simulation, Chem. Phys. Lett. 223 (1994), 411-415. 
33) M. Boero, K. Terakura, T. Ikeshoji, C. C. Liew, M. Parinello, Water at supercritical conditions: A first principles study, J. Chem. Phys. 115 (2001), 22192227.

34) A. A. Chialvo, P. T. Cummings, Microstructure of Ambient and Supercritical Water. Direct Comparison between Simulation and Neutron Scattering Experiments, J. Phys. Chem. 100 (1996), 1309-1316.

35) Y. Guissani, B. Guillot, A computer simulation study of the liquid-vapor coexistence curve of water, J. Chem. Phys. 98 (1993), 8221-8235.

36) J. Alejandre, D. J. Tildesley, Molecular dynamics simulation of the orthobaric densities and surface tension of water, J. Chem. Phys. 102 (1994), 4574-4583.

37) R. D. Mountain, Comparison of a fixed-charge and a polarizable water model, J. Chem. Phys. 103 (1995), 3084-3090.

38) R. D. Mountain, Voids and clusters in expended water, J. Chem. Phys. 110 (1999), 2109-2115.

39) F. Lafrad, T. Tassaing, M. Kiselev, A. Idrissi, The local structure of sub- and supercritical water as studied by FTIR spectroscopy and molecular dynamics simulations, J. Mol. Liq. 239 (2017), 61-67.

40) M. Boero, K. Terakura, T. Ikeshoji, C.C. Liew, M. Parrinello, Hydrogen Bonding and Dipole Moment of Water at Supercritical Conditions: A First-Principles Molecular Dynamics Study, Phys. Rev. Lett. 85 (2000), 3245-3248.

41) H. Ma, J. Ma, Density dependence of hydrogen bonding and the translationalorientational structural order in supercritical water: A molecular dynamics study, J. Chem. Phys. 135 (2011), 054504.

42) I. Skarmoutsos, J. Samios, Local Density Inhomogeneities and Dynamics in Supercritical Water: A Molecular Dynamics Simulation Approach, J. Phys. Chem. B 110 (2006), 21931-21937.

43) I. Skarmoutsos, J. Samios, Local density augmentation and dynamic properties of hydrogen- and non-hydrogen bonded supercritical fluids: A molecular dynamics study, J. Chem. Phys. 126 (2007), 044503. 
44) I. Skarmoutsos, E. Guardia, Local Structural Effects and Related Dynamics in Supercritical Ethanol. 1. Mechanisms of Local Density Reorganization and Residence Dynamics, J. Phys. Chem. B 113 (2009), 8887-8897.

45) S.C. Tucker, Solvent Density Inhomogeneities in Supercritical Fluids, Chem. Rev. 99 (1999), 391-418.

46) J. M. Stubbs, Molecular simulations of supercritical fluid systems, J. Supercrit. Fluids 108 (2016), 104-122.

47) W. Smith, T.R. Forester, DL_POLY_2.0: A general-purpose parallel molecular dynamics simulation package, J. Mol. Graph. 14 (1996), 136-141.

48) L. Martínez, R. Andrade, E.G. Birgin, J.M. Martínez, PACKMOL: A Package for Building Initial Configurations for Molecular Dynamics Simulations, J. Comput. Chem. 30 (2009), 2157-2164.

49) W. G. Hoover, Canonical dynamics: Equilibrium phase-space distributions, Phys. Rev. A 31 (1985), 1695-1697.

50) J. P. Ryckaert, G. Ciccotti, H. J. C. Berendsen, Numerical integration of the Cartesian Equations of Motion of a System with Constraints: Molecular Dynamics of n-Alkanes, J. Comp. Phys. 23 (1977), 327-341.

51) H. J. C. Berendsen, J. R. Grigera, T. P. Straatsma, The missing term in effective pair potentials, J. Phys. Chem. 91 (1987), 6269-6271.

52) E. Guardia, J. Marti, Density and temperature effects on the orientational and dielectric properties of supercritical water, Phys. Rev. E 69 (2004), 011502.

53) E. Johansson, K. Bolton, P. Ahlström, Simulations of vapor water clusters at vapor-liquid equilibrium, J. Chem. Phys. 123 (2005), 024504.

54) E. Guardia, D. Laria, J. Marti, Hydrogen Bond Structure and Dynamics in Aqueous Electrolytes at Ambient and Supercritical Conditions, J. Phys. Chem. B 110 (2006), 6332-6338. 
55) W. Song, R. Biswas, M. Maroncelli, Intermolecular Interactions and Local Density Augmentation in Supercritical Solvation: A Survey of Simulation and Experimental Results, J. Phys. Chem. A 104 (2000), 6924-6939.

56) W. Song, M. Maroncelli, Local density augmentation in neat supercritical fluids: the role of electrostatic interactions, Chem. Phys. Lett. 378 (2003), 410-419.

57) S. Nugent, B. M. Ladanyi, The effects of solute-solvent electrostatic interactions on solvatochromic shifts in supercritical $\mathrm{CO}_{2}$, J. Chem. Phys. 120 (2003), 874-884.

58) J. R. Errington, P.G. Debenedetti, Relationship between structural order and the anomalies of liquid water, Nature 409 (2001), 318-321.

59) C. Zhang, L. Spanu, G. Galli, Entropy of Liquid Water from Ab Initio Molecular Dynamics, J. Phys. Chem. B 115 (2011), 14190-14195.

60) H. Ma, Density dependence of the entropy and the solvation shell structure in supercritical water via molecular dynamics simulation, J. Chem. Phys. 136 (2012), 214501.

61) R. H. Henchman, S. J. Cockram, Water's non-tetrahedral side, Faraday Discuss., 167 (2013), 529-550.

62) I. Skarmoutsos, M. Masia, E. Guardia, Structural and dipolar fluctuations in liquid water: A Car-Parrinello molecular dynamics study, Chem. Phys. Lett. 648 (2016), 102-108.

63) E. Guardia, I. Skarmoutsos, M. Masia, Hydrogen Bonding and Related Properties in Liquid Water: A Car-Parrinello Molecular Dynamics Simulation Study, J. Phys. Chem. B 119 (2015), 8926-8938.

64) A. Idrissi, P. Damay, M. Kiselev, Nearest neighbor assessments of spatial distribution in CO2: A molecular dynamics analysis, Chem. Phys. 332 (2007), 139143.

65) I. Skarmoutsos, S. Mossa, J. Samios, Structure and dynamics of liquid $\mathrm{CS}_{2}$ : Going from ambient to elevated pressure conditions, J. Chem. Phys. 145 (2016), 154505.

66) B.I. Sedunov, Structural Transitions in Supercritical Fluids, Journal of Thermodynamics (2011), ID: 194353 
67) G.G. Simeoni, T. Bryk, F.A. Gorelli, M. Krisch, G. Ruocco, M. Santoro, T. Scopigno, The Widom line as the crossover between liquid-like and gas-like behaviour in supercritical fluids, Nature Physics 6 (2010), 503-507

\section{TABLES}

Table I: Simulated thermodynamic state points of supercritical water, along the supercritical isotherm of $\mathrm{T}=666 \mathrm{~K}$.

\begin{tabular}{|c|c|}
\hline $\begin{array}{c}\text { Simulated State } \\
\text { Points }\end{array}$ & Density $\left(\mathrm{g} / \mathrm{cm}^{3}\right)$ \\
\hline 1 & 0.0322 \\
\hline 2 & 0.0644 \\
\hline 3 & 0.1288 \\
\hline 4 & 0.1932 \\
\hline 5 & 0.2576 \\
\hline 6 & 0.322 \\
\hline 7 & 0.3864 \\
\hline 8 & 0.4508 \\
\hline 9 & 0.5152 \\
\hline 10 & 0.5796 \\
\hline 11 & 0.644 \\
\hline
\end{tabular}




\section{FIGURES}

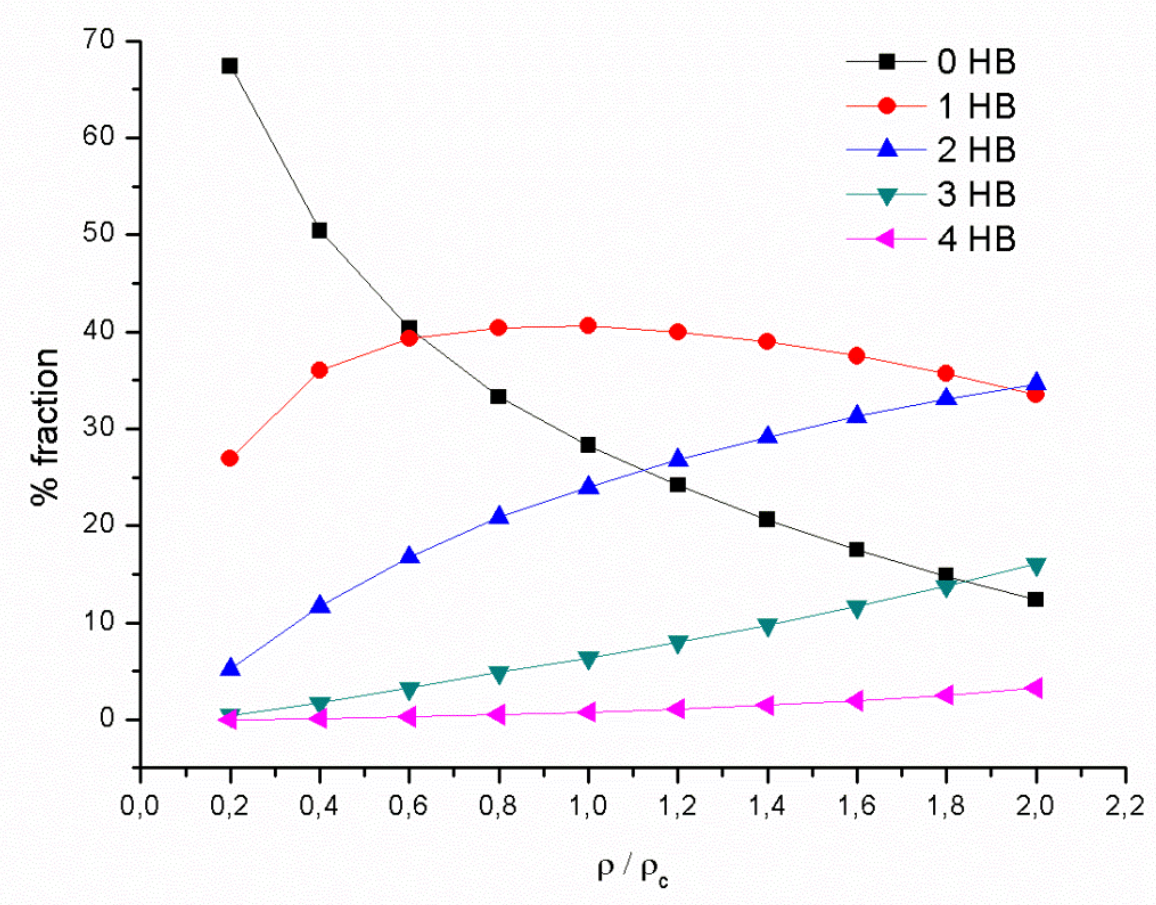

Figure 1 

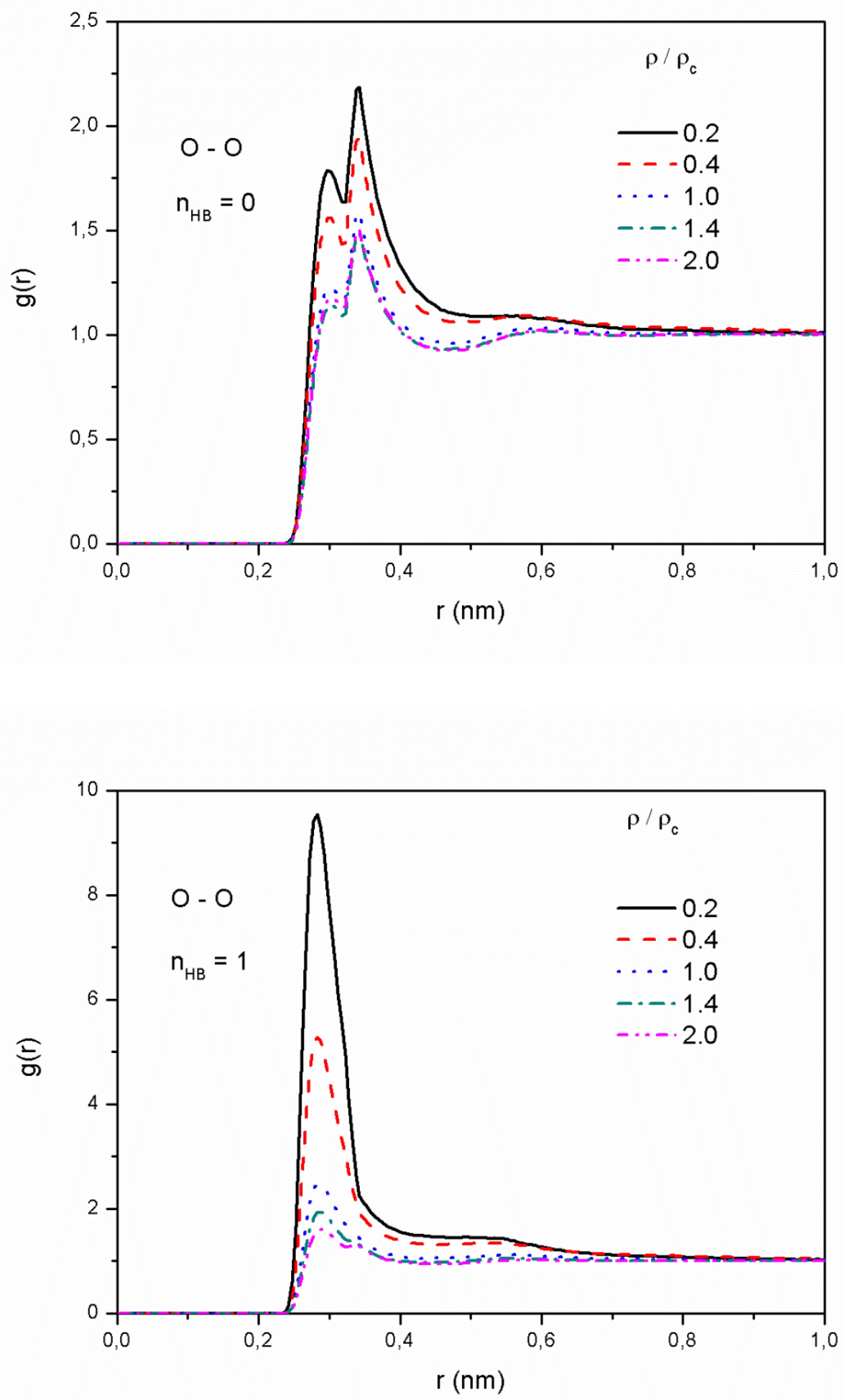

Figure 2 

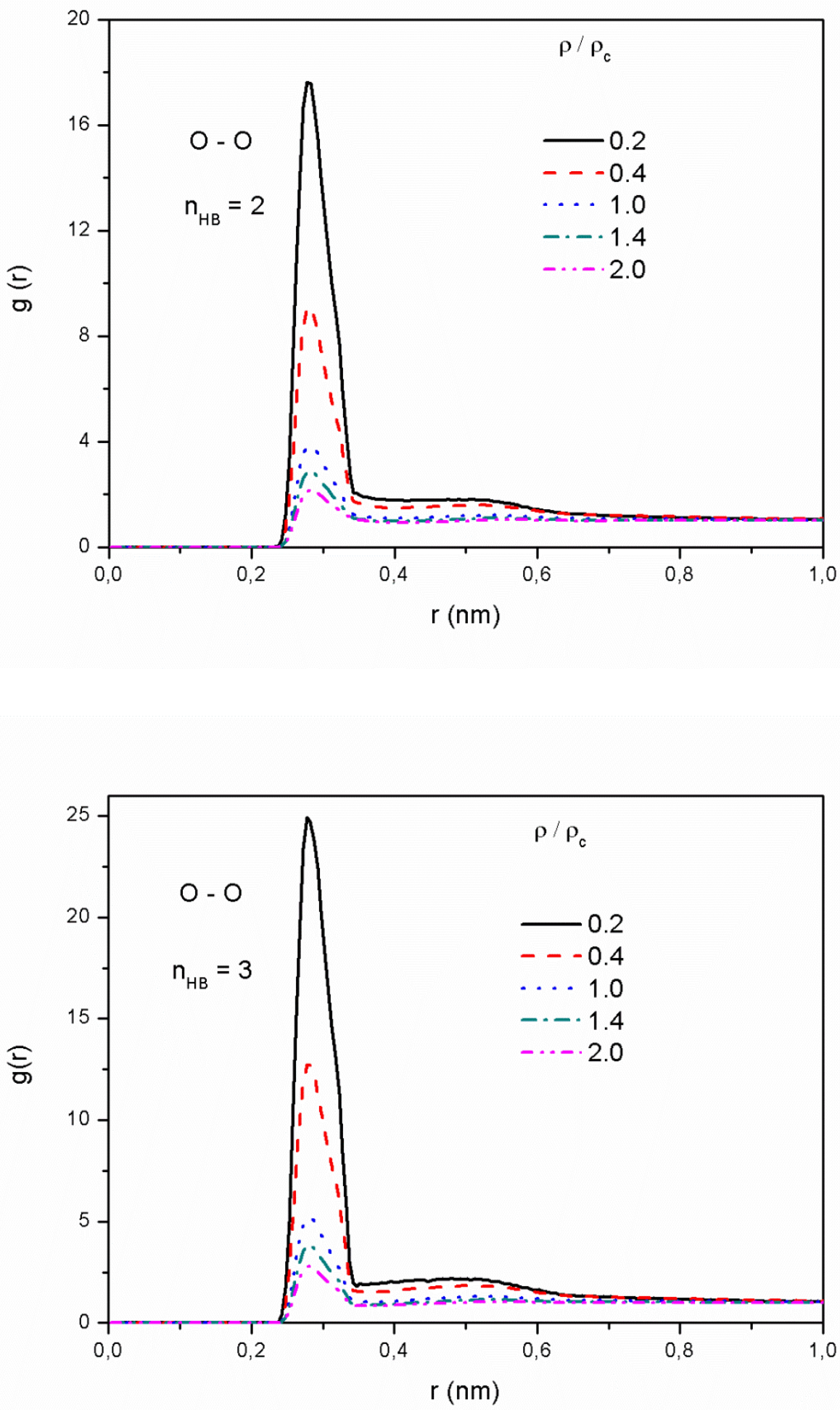

Figure 3 

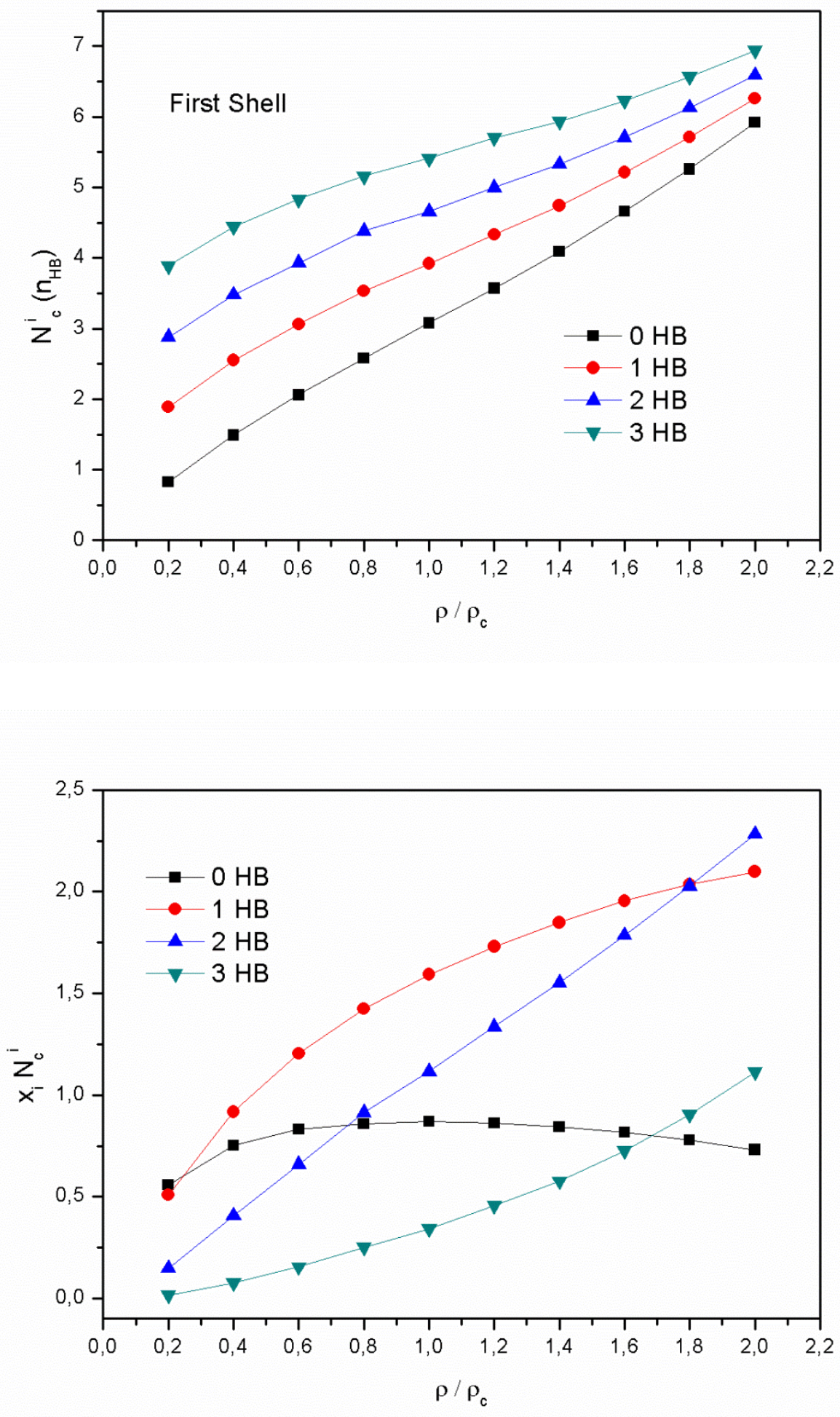

Figure 4 


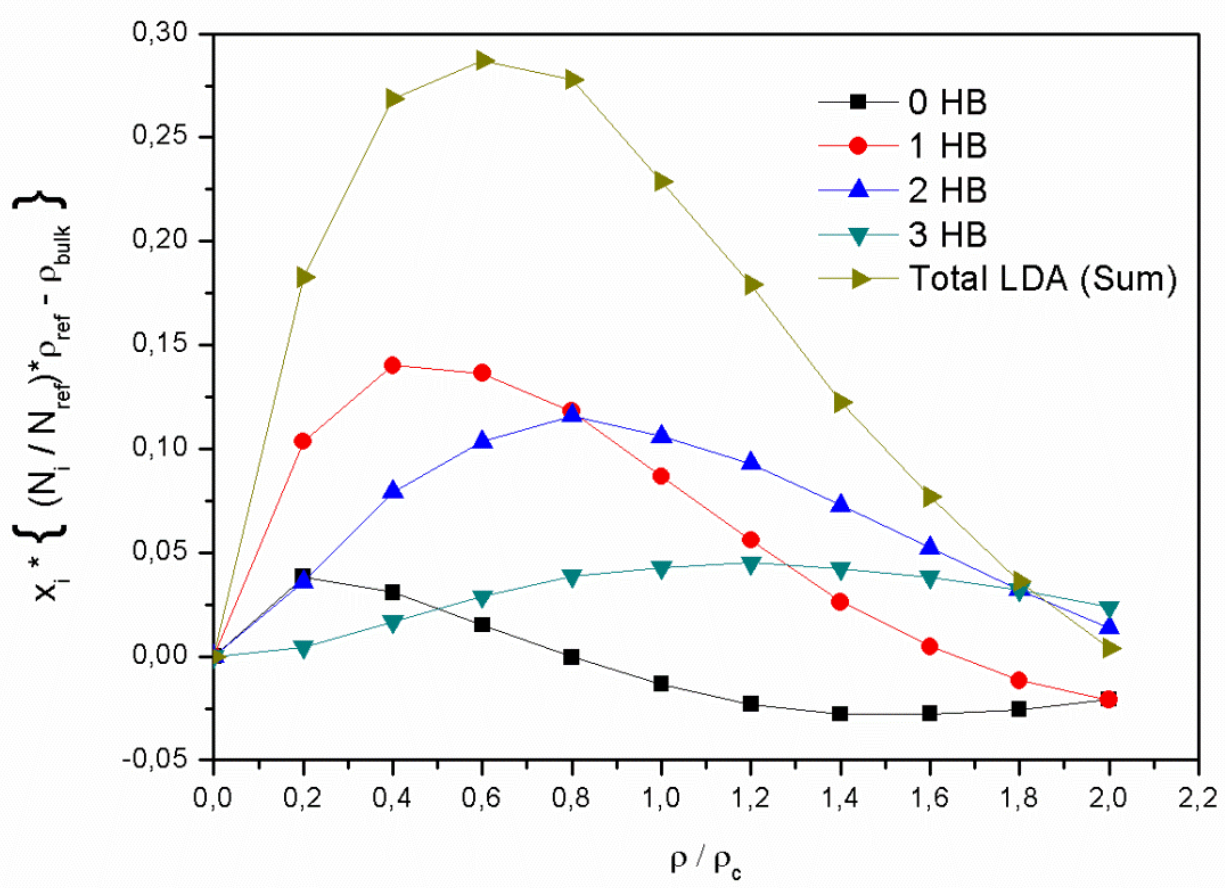

Figure 5 

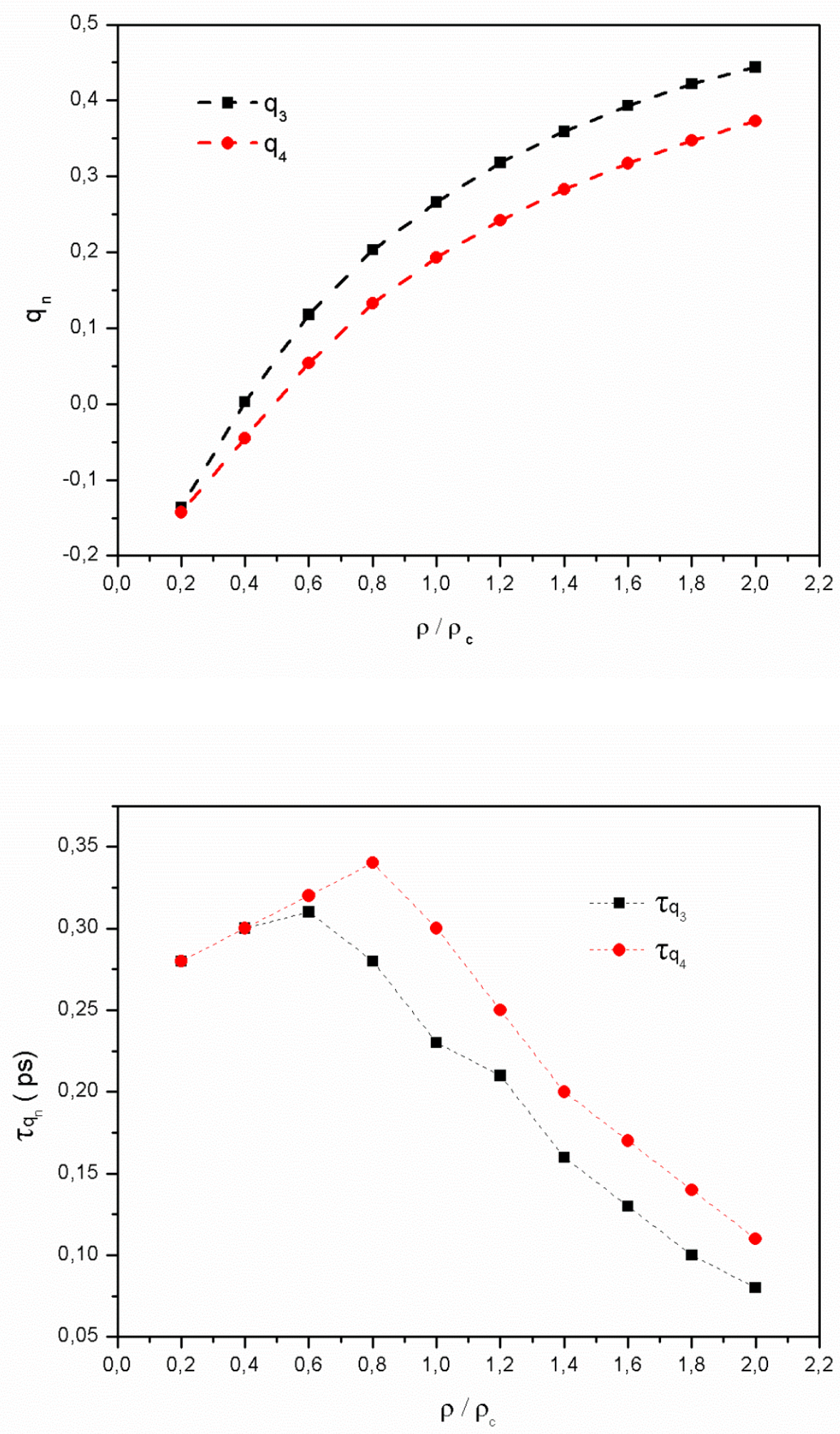

Figure 6 

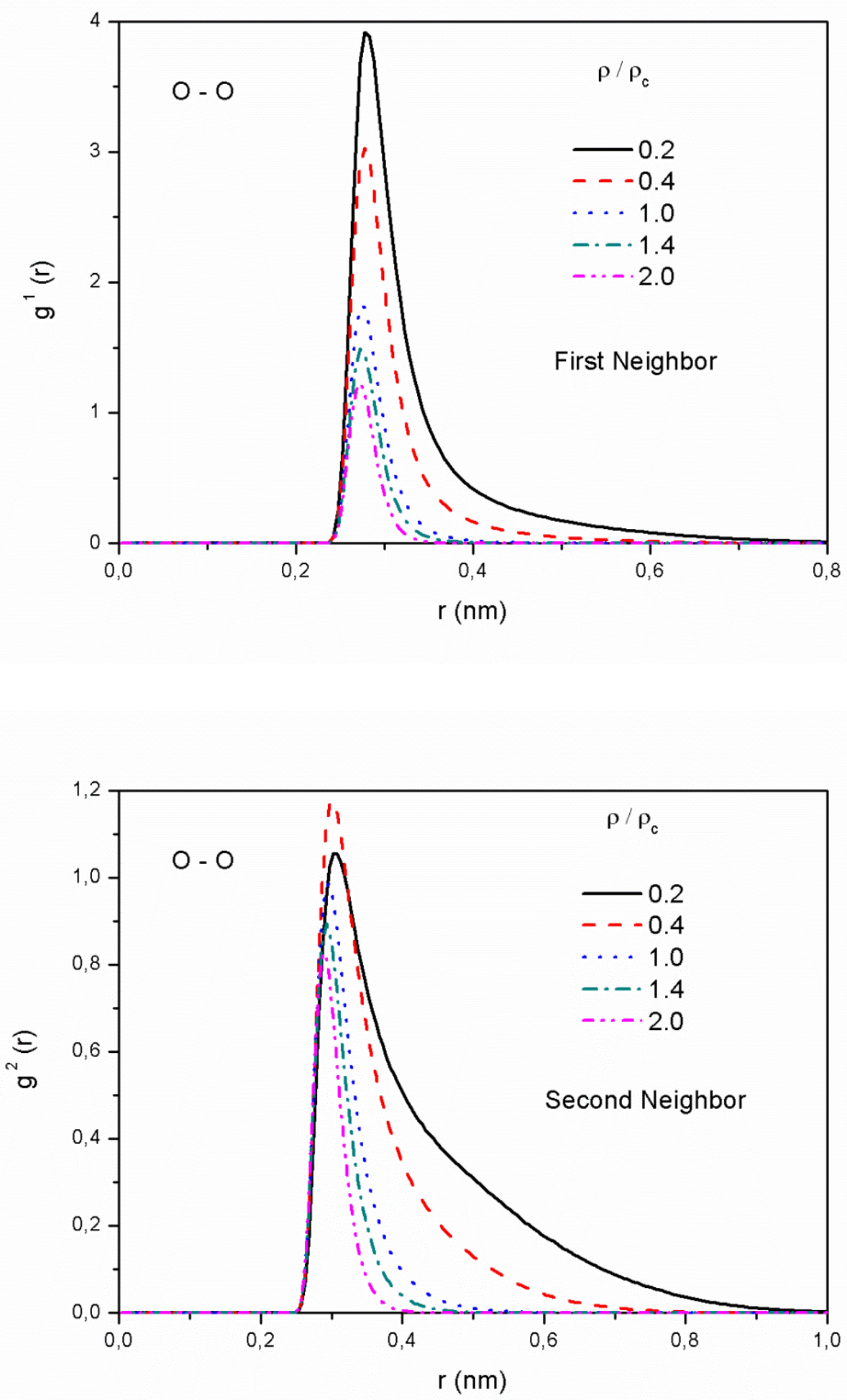

Figure 7 

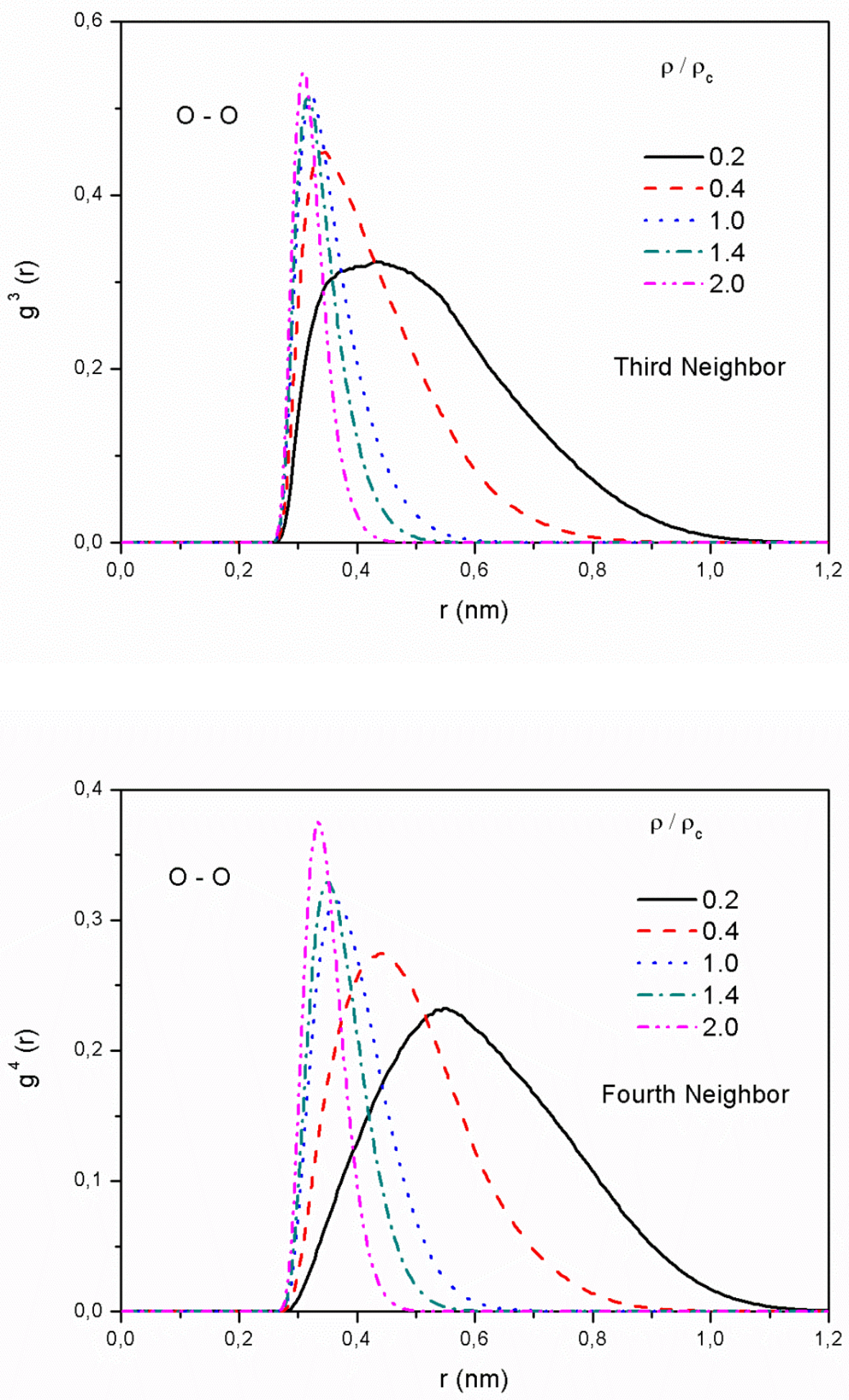

Figure 8 

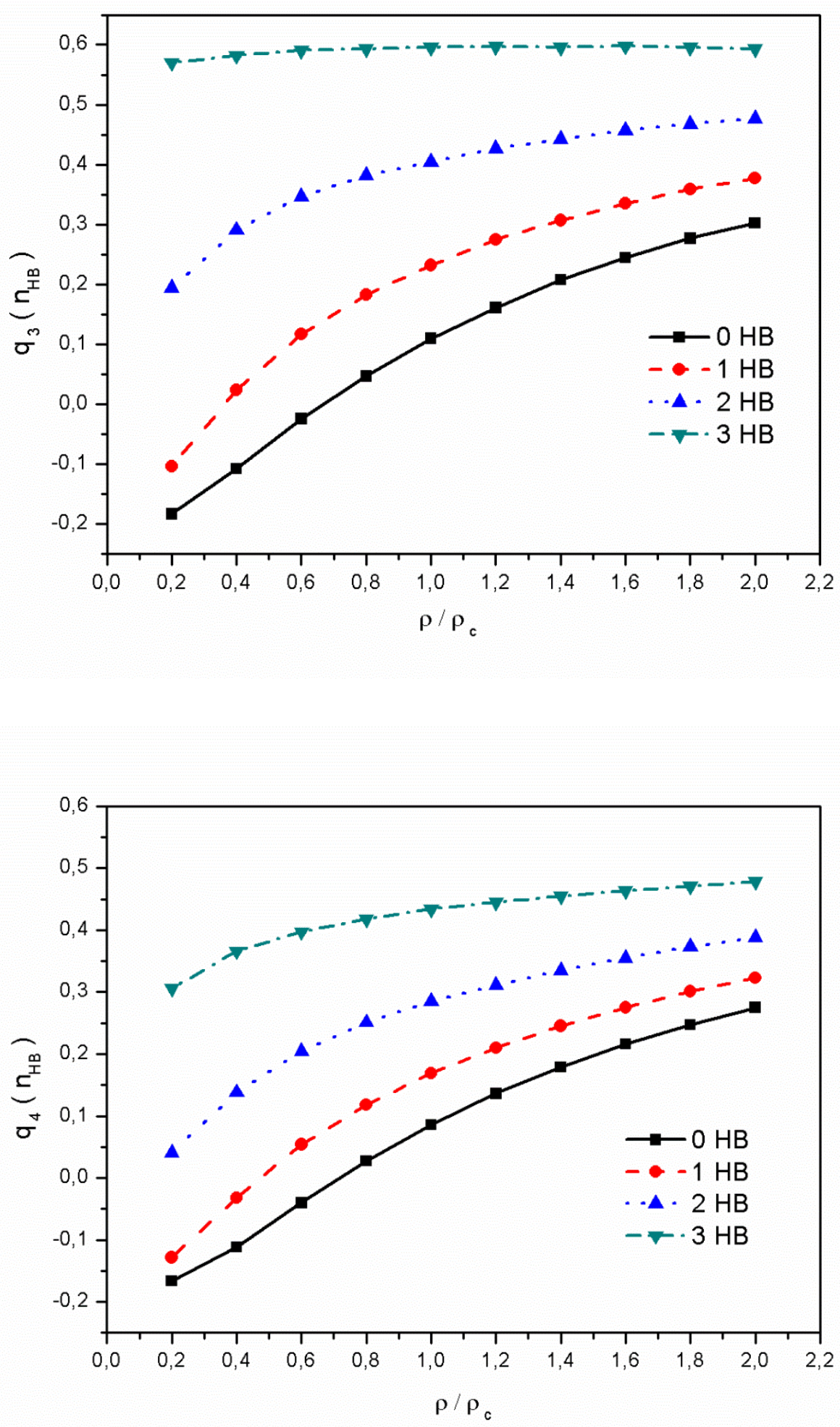

Figure 9 


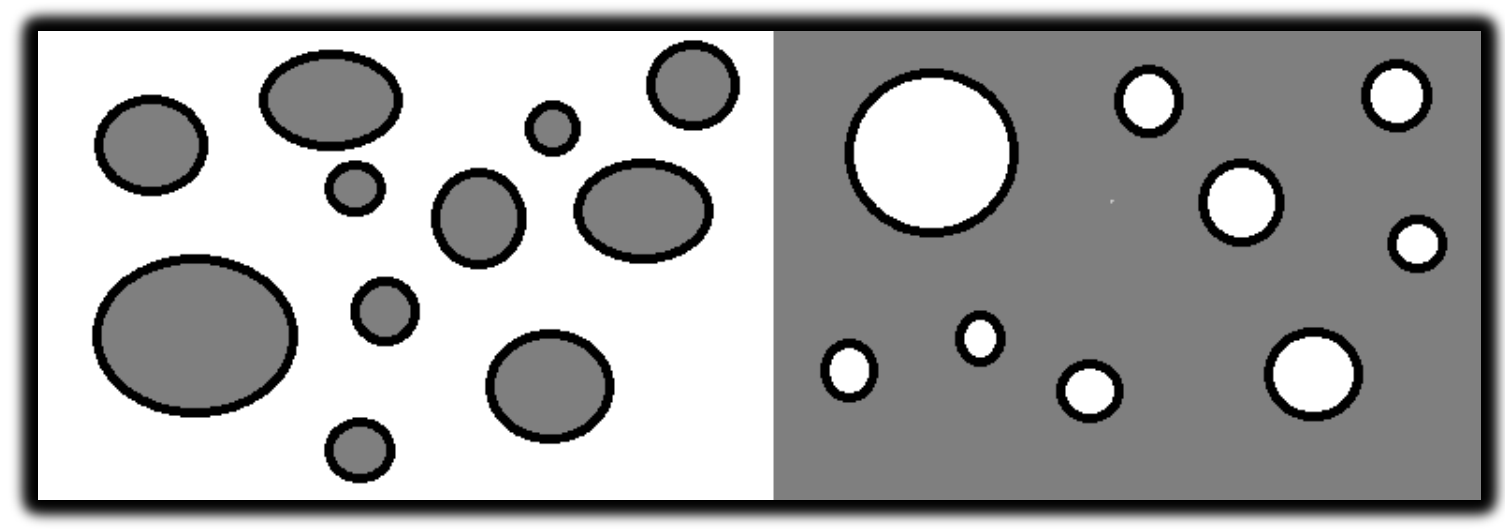

Figure 10 

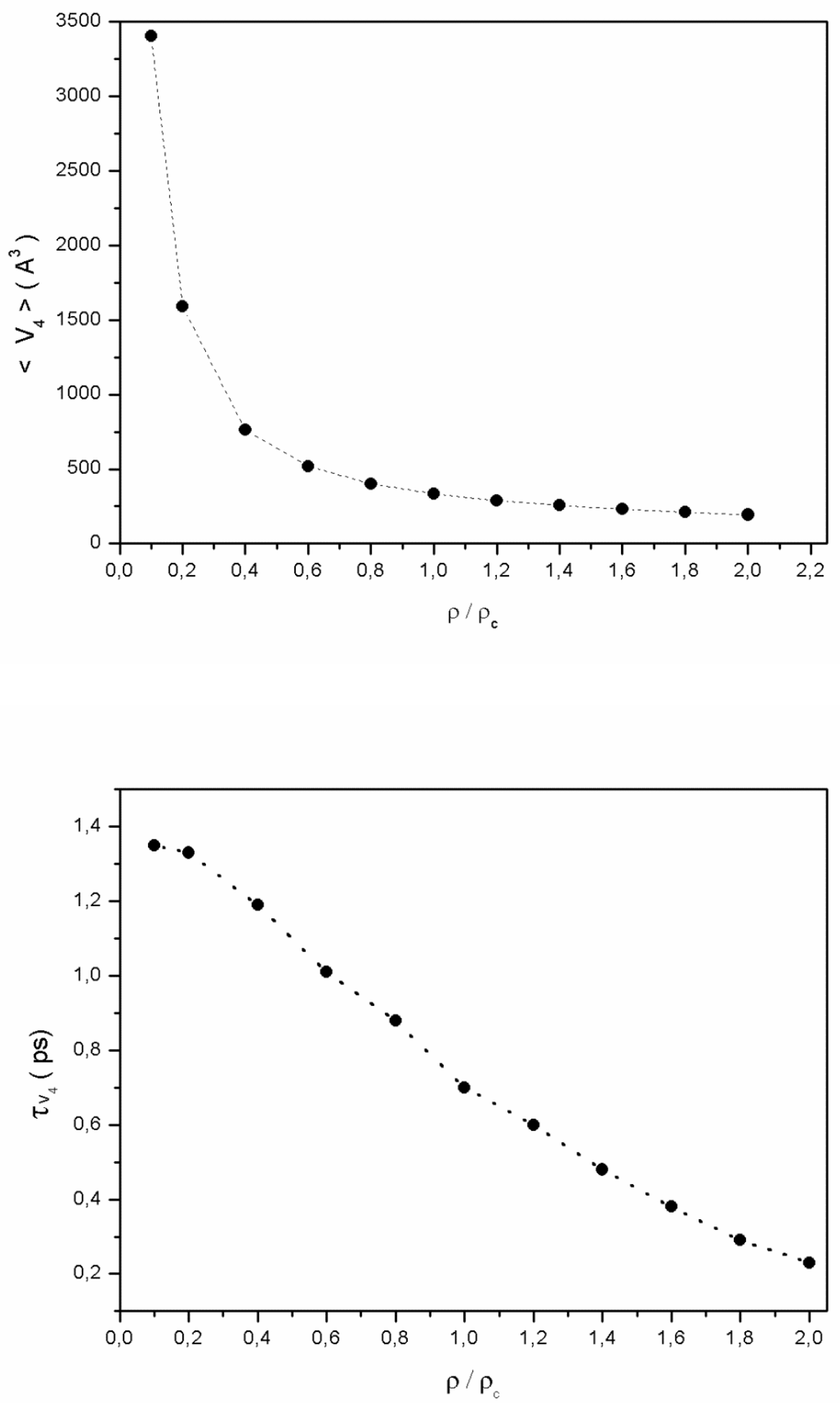

Figure 11 


\section{FIGURE CAPTIONS}

Figure 1: Calculated fractions of water molecules forming 0-4 hydrogen bonds as a function of the bulk density of SCW.

Figure 2: Bulk density dependence of the O-O rdf for water molecules forming zero and one hydrogen bonds.

Figure 3: Bulk density dependence of the O-O rdf for water molecules forming two and three hydrogen bonds.

Figure 4: Bulk density dependence of the coordination number for water molecules forming $\mathrm{i}=0-3$ hydrogen bonds and of their contributions $\chi_{i} \cdot N_{c}^{i}$ to the total average coordination number.

Figure 5: The contributions of water molecules forming 0-3 hydrogen bonds to the overall average LDA as a function of the bulk density.

Figure 6: Bulk density dependence of the average orientational order parameters $q_{3}$ and $q_{4}$, as well as of their corresponding relaxation times $\tau_{q_{3}}$ and $\tau_{q_{4}}$.

Figure 7: The contributions of the first and second nearest neighbors to the overall O-O rdfs for representative bulk densities in terms of the functions $g^{1}(r)$ and $g^{2}(r)$.

Figure 8: The contributions of the third and fourth nearest neighbors to the overall O-O rdfs for representative bulk densities in terms of the functions $g^{3}(r)$ and $g^{4}(r)$.

Figure 9: Bulk density dependence of the orientational order parameters $q_{3}$ and $q_{4}$ for water molecules forming 0-3 hydrogen bonds.

Figure 10: Schematic representation of the gas-like (left) and liquid-like (right) structure of water at near-critical, supercritical temperatures. The gas-like fluid consists of small isolated clusters interacting like gas-phase particles inside a vacuum environment. The liquid-like fluid structure is similar to a continuous condensed medium consisted of a distribution of voids and regions of different density, resembling an inhomogeneous porous liquid. 
Figure 11: Bulk density dependence of the calculated average volume $V_{4}$ of the sphere containing the four nearest neighbors of each water molecule and its corresponding correlation time $\tau_{V_{4}}$. 